\title{
Revision surgery for incomplete resection or recurrence of cervical spine chordoma: a consecutive case series of 24 patients
}

\author{
Peter Obid ${ }^{1,2}$ (D) Tamás Fekete ${ }^{1} \cdot$ Philipp Drees $^{3} \cdot$ Daniel Haschtmann $^{1} \cdot$ Frank Kleinstück $^{1} \cdot$ Markus Loibl $^{1}$. \\ Dezsö Jeszenszky ${ }^{1}$
}

Received: 17 December 2020 / Revised: 25 May 2021 / Accepted: 23 July 2021 / Published online: 2 August 2021

(c) The Author(s) 2021

\begin{abstract}
Purpose Chordomas are rare tumors with an annual incidence of approximately one per million. Chordomas rarely metastasize but show a high local recurrence rate. Therefore, these patients present a major clinical challenge, and there is a paucity of the literature regarding the outcome after revision surgery of cervical spine chordomas. Available studies suggest a significantly worse outcome in revision scenarios. The purpose of this study is to analyze the survival rate, and complications of patients that underwent revision surgery for local recurrence or incomplete resection of chordoma at the craniocervical junction or at the cervical spine.

Methods 24 consecutive patients that underwent revision surgery for cervical spine chordoma remnants or recurrence at a single center were reviewed retrospectively. We analyzed patient-specific surgical treatment strategies, complications, and outcome. Kaplan-Meier estimator was used to analyze five-year overall survival.

Results Gross total resection was achieved in 17 cases. Seven patients developed dehiscence of the pharyngeal wall, being the most common long-term complication. No instability was observed. Postoperatively, four patients received proton beam radiotherapy and 12 patients had combined photon and proton beam radiotherapy. The five-year overall survival rate was $72.6 \%$.

Conclusion With thorough preoperative planning, appropriate surgical techniques, and the addition of adjuvant radiotherapy, results similar to those in primary surgery can be achieved.
\end{abstract}

Keywords Chordoma $\cdot$ Recurrence and revision surgery $\cdot$ Cervical spine and craniocervical junction $\cdot$ Outcome and complications $\cdot$ Survival

\section{Introduction}

Chordomas are malignant bone tumors which typically occur in adolescents or young and middle-aged adults and were probably first described by Rudolf Virchow in 1857 [1]. Chordomas are rare tumors with an annual incidence of approximately one per million. They account for $1 \%$ to $8 \%$ of

Peter Obid

p.obid@gmx.de

1 Department of Spine Surgery, Schulthess Klinik, Zürich, Switzerland

2 Department of Orthopaedics and Orthopaedic Surgery, Greifswald University Hospital, Ferdinand-Sauerbruch-Stras se, 17475 Greifswald, Germany

3 Department of Orthopaedics and Traumatology, Mainz University Hospital, Mainz, Germany primary malignant bone tumors and for $20 \%$ of those arising from the spine [2]. Chordomas usually show a low-grade but locally invasive malignancy and are life-threatening without appropriate treatment [3]. Growth of these tumors is usually slow, and they infiltrate local bone and adjacent tissues with a high rate of local recurrence. Additionally, these tumors are refractory to conventional radiotherapy or chemotherapy. Metastases rarely occur, typically at a late stage of the disease and usually after evidence of local recurrence. Overall, the effect of the disease is more a function of its local aggressiveness than its potential to metastasize [4].

Generally, en bloc resection with wide or marginal margins is the treatment of choice for chordomas, especially when they are located at the thoracic or lumbar spine [5, 6]. En bloc resections of chordomas located at the cervical spine or at the craniocervical junction pose special challenges. The anatomical proximity of vital structures including the 
spinal cord, nerve roots, esophagus, and vertebral arteries makes wide margin en bloc resection highly difficult. There are only smaller case series or case reports of en bloc resection of cervical spine chordomas [7, 8]. These resections usually involve the sacrifice of cervical nerve roots and are associated with a high rate of morbidity and mortality. It is logical to assume that en bloc resection provides a superior outcome; however, high-quality evidence is missing. Larger case series of non-en bloc gross total resection for cervical spine chordoma show an improved five- and ten-year overall survival as well as an improved quality of life after surgery $[9,10]$.

The available data focus on the outcome after primary surgery for chordoma of the craniocervical junction and cervical spine. Five-year overall survival rates of $62 \%$ to $85.7 \%$ could be achieved [2,10-13]. Complete tumor removal is the crucial factor for outcome. The chance of long-term survival after local recurrence is low, and local control is rarely achieved [14]. Although local recurrence is common following surgical treatment of spinal chordoma, there is only very limited data available that analyze the outcome after revision surgery for cervical spine chordoma recurrence or remnants. The aim of this study is to provide further data and to analyze the outcome and complications of 24 consecutive patients that underwent revision surgery for recurrence or remnants of cervical spine chordoma.

\section{Methods}

We retrospectively reviewed all patients that underwent revision surgery for recurrence or remnants of chordoma at the craniocervical junction or at the cervical spine (without associated intracranial involvement) between 1999 and 2014. 24 patients could be included. Data were collected from our department's medical records. The patient collective was international (Australia $n=1$, Austria $n=3$, Canada $n=1$, Germany $n=2$, Greece $n=1$, Hungary $n=1$, Israel $n=1$, Italy $n=2$, Russia $n=1$, Switzerland $n=6$, USA $n=5$ ) which made acquisition of mid- and long-term postoperative data very challenging. Therefore, we were not able to acquire complete follow-up data of three patients. These patients were not included in survival calculations. We analyzed the patients' basic demographics, the time between primary and revision surgery, five-year overall survival rate, complications, type of surgery at primary surgery and at revision surgery, and type of instrumentation. Kaplan-Meier estimator was used to analyze five-year overall survival.

If the first magnetic resonance imaging (MRI) scan after the initial surgery did not clearly show tumor remnants, it was classified as recurrence; otherwise, it was classified as remnant. If no MRI scan was performed postoperatively, we classified the tumor as remnant. All patients have undergone at least one surgery before they have been referred to our department.

Preoperatively, all patients received plain radiographs, computed tomography (CT) scan including three-dimensional angiography, and MRI scan. Particular interest was paid to the extent of the tumor, signs of spinal instability, and encasement or displacement of vertebral arteries (VA). CT angiography and duplex sonography were used to evaluate dominance, hypoplasia, and variations of vertebral arteries. Difference in the diameter of the VAs of $\geq 1 \mathrm{~mm}$, or asymmetry in the merging of the two VAs to the vertebrobasilar junction was defined as criteria for VA dominance [15]. A balloon occlusion test was performed if a dominant or codominant VA was located within or close to the tumor.

Intraoperatively, complete tumor removal and clear margins were identified with the assistance of the surgical microscope. All tissue that was in contact with the tumor (including the dura and VAs) was devitalized superficially using bipolar coagulation.

\section{Results}

\section{Surgery}

17 tumors were located at the craniocervical junction, and 7 were located at the subaxial cervical spine. Figure 1 provides an overview of the localization of the tumors and differentiates between recurrence and remnant. If the postoperative MRI showed incomplete resection, this was interpreted as tumor remnant. 34 operations were performed before admittance to our department (Table 1). The primary surgeries were without exception piecemeal, intralesional or partial resections with or without instrumentation of the spine. Another 50 operations were performed at our department (Table 2). Mean duration of surgeries was $451.6 \mathrm{~min}$ ( \pm 298.5$)$. Mean blood loss was $1670.3 \mathrm{ml}( \pm 2.527 .7)$. The average time between the latest surgery and admittance to our department was 4 months (range: $2-6$ ) for remnant chordoma, and 12.1 months (range: 4-21) for recurrence chordoma (Table 3).

All patients underwent single- or multi-staged tumor removal combined with revision of instrumentation or primary instrumentation and spondylodesis. Tables 4 and 5 provide an overview of the approaches and the type of instrumentation used in revision surgery. Iliac and fibular grafts were used for spondylodesis, Harms cages and $\mathrm{C} 2$ prostheses were used for anterior instrumentation, and screw/rod constructs were used for posterior instrumentation [16]. One patient was instrumented to the clivus using a modified $\mathrm{C} 2$ prosthesis that was fixed to the clivus with a screw. A vertebral artery was resected in 10 cases (Table 3 ). Table 2 shows the number of surgeries per patient. Multiple 
Fig. 1 Localization of tumor remnant or recurrence

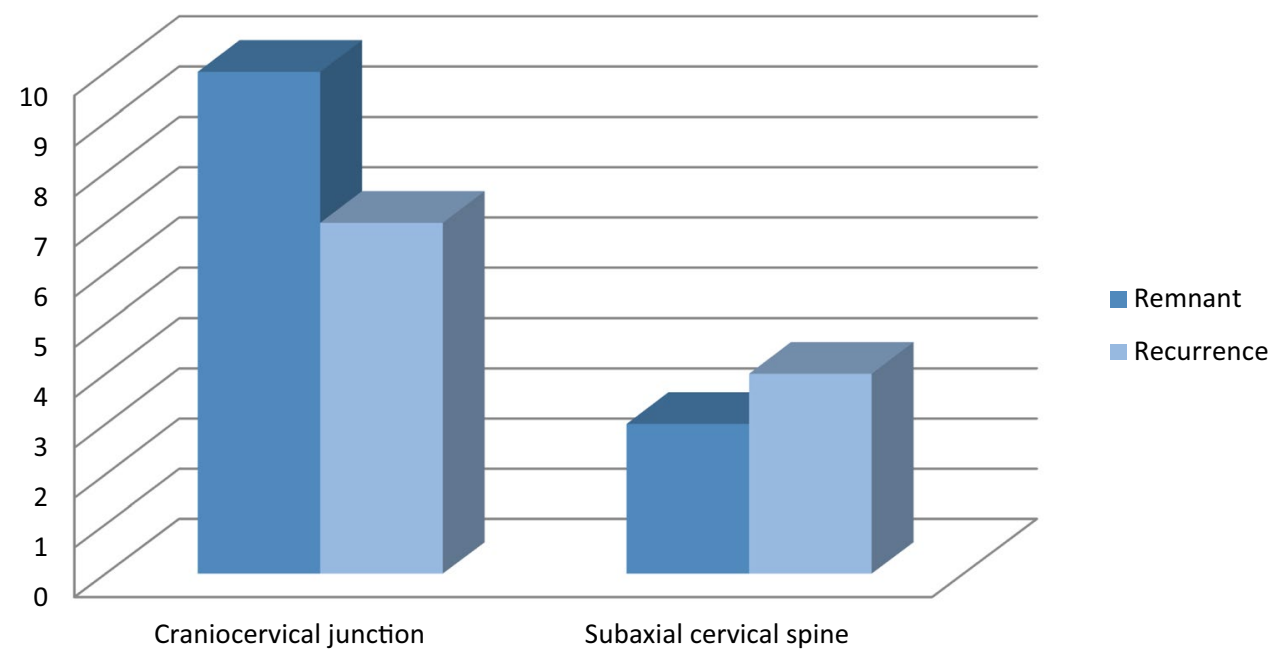

Table 1 Primary surgeries

\begin{tabular}{lcl}
\hline & No. of patients & Implants \\
\hline No instrumentation & 10 & \\
Anterior & 5 & $2 \times$ Plate \\
& & $2 \times$ Fibular graft and plate \\
& & $1 \times$ Iliac graft and plate \\
& 3 & Screws and rods \\
Posterior & 4 & Various \\
Combined & &
\end{tabular}

Table 2 Number of revision surgeries per patient

surgeries were not only necessary due to complications but also due to incomplete tumor removal. Nine patients could be treated with one surgery. One patient underwent seven surgeries.

\section{Patient collective}

12 patients were female, and 12 were male (Table 6). Of those, 5 patients were children ( 4 male and 1 female). Mean age at surgery was 53.4 years $( \pm 21.9)$. Mean follow-up was 54.5 months $( \pm 55.42)$.

Complete follow-up data were available for 21 patients. Based on postoperative imaging, gross total tumor removal was achieved in 17 patients, and a subtotal resection was performed in three patients. One palliative surgery with decompression only was performed in a patient that presented with pulmonary metastases.

New permanent neurological symptoms did not occur. Four patients presented with a preexisting myelopathy. One patient developed a temporary hemiparesis. However, complications were not uncommon. Three patients developed a clinically relevant hematoma and required revision. Seven cerebrospinal fluid (CSF) leaks occurred intraoperatively, and one of the patients developed meningitis. All leaks were closed intraoperatively either by direct closure or by performing a duraplasty with either autologous tissue or allograft material (e.g., artificial dura, fibrin glue, dermal fat graft, or flaps of nasal septal mucosa) [17].

All patients with an intraoperative CSF leak received a continuous lumbar drainage for five days. Dehiscence of the pharyngeal wall at the sight of incision was the most common long-term complication, and it occurred in seven patients. These patients required multiple revisions. Six patients received a radial forearm flap, and one patient received a free jejunal interposition graft. Two patients underwent early revision due to incomplete resection of the tumor. No instability was observed. Postoperatively, all patients received ergotherapy and speech therapy. Severe dysphagia has been treated with a temporary PEG tube and parenteral nutrition.

16 patients received postoperative proton beam or proton/photon beam radiotherapy earliest after three months postoperatively to rule out any serious complication (Fig. 2). We could not identify any statistically significant differences between the recurrence group and the remnant group. The 5-year progression-free survival rate was $72.6 \%$ (Fig. 3). 
Table 3 Dominance and resection of vertebral arteries, tumor histology
Table 4 Approaches and instrumentation used in revision surgery

Table 5 Anterior and posterior implants used for revision surgeries

\begin{tabular}{|c|c|c|c|c|}
\hline Patient & Dominance of VA & Resection of VA & $\begin{array}{l}\text { Contact of tumor } \\
\text { with VA }\end{array}$ & $\begin{array}{l}\text { Histological } \\
\text { subtype of the } \\
\text { tumor }\end{array}$ \\
\hline \#1 & Co-Dominance & Left VA & Left VA & $\mathrm{n} / \mathrm{a}$ \\
\hline$\# 2$ & Co-Dominance & None & Right VA & Conventional \\
\hline \#3 & Co-Dominance & Left VA & Left VA & Chondroid \\
\hline \#4 & $\mathrm{n} / \mathrm{a}$ & None & None & Conventional \\
\hline \#5 & Left VA & Right VA & Right VA & $\mathrm{n} / \mathrm{a}$ \\
\hline \#6 & Co-Dominance & None & Both & $\mathrm{n} / \mathrm{a}$ \\
\hline \#7 & Co-Dominance & None & Both & Conventional \\
\hline \#8 & Co-Dominance & None & Both & Conventional \\
\hline \#9 & Co-Dominance & None & None & Conventional \\
\hline \#10 & Left VA & Right VA & Both & Conventional \\
\hline \#11 & Co-Dominance & Left VA & Both & Conventional \\
\hline \#12 & Co-Dominance & Left VA & Left VA & Conventional \\
\hline \#13 & Co-Dominance & Left VA & Left VA & Conventional \\
\hline$\# 14$ & Co-Dominance & None & Right VA & Chondroid \\
\hline \#15 & Co-Dominance & None & Left VA & Conventional \\
\hline \#16 & Right VA & None & Left VA & Conventional \\
\hline \#17 & Co-Dominance & None & Right VA & Conventional \\
\hline \#18 & Co-Dominance & None & Right VA & Dedifferentiated \\
\hline \#19 & Co-Dominance & Left VA & Left VA & Conventional \\
\hline \#20 & Co-Dominance & Left VA & Both & Conventional \\
\hline \#21 & Right VA & None & Both & Chondroid \\
\hline \#22 & Co-Dominance & None & None & Conventional \\
\hline \#23 & Co-Dominance & None & Both & Conventional \\
\hline \#24 & Right VA & Left VA & Left VA & Conventional \\
\hline
\end{tabular}

$V A$ Vertebral artery, n/a data not available

\begin{tabular}{|c|c|c|c|}
\hline & & & No. of patients \\
\hline \multirow[t]{4}{*}{ Approach } & \multicolumn{2}{|c|}{ Anterior midline/posterior } & 12 \\
\hline & \multicolumn{2}{|c|}{ Posterior/anterior midline/transoral } & 7 \\
\hline & \multicolumn{2}{|c|}{ Posterior/transoral } & 3 \\
\hline & \multicolumn{2}{|l|}{ Anterolateral } & 2 \\
\hline \multirow[t]{4}{*}{ Instrumentation } & \multicolumn{2}{|c|}{ No instrumentation } & 1 (palliative surgery) \\
\hline & \multicolumn{2}{|l|}{ Anterior } & 1 \\
\hline & \multicolumn{2}{|l|}{ Posterior } & 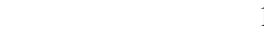 \\
\hline & \multicolumn{2}{|c|}{ Anterior/posterior } & 21 \\
\hline Anterior Instrumentation & No. of patients & Posterior Instrumentation & No. of patients \\
\hline Iliac graft + plate & 5 & Screws + rods & 14 \\
\hline Fibular graft + plate & 1 & Iliac graft + screws + rods & 3 \\
\hline C2-prosthesis & 11 & & \\
\hline Harms cage + plate & 2 & & \\
\hline
\end{tabular}


Table 6 Basic patient demographics

\begin{tabular}{llll}
\hline & Adult & Pediatric & Total \\
\hline Sex & $8 \mathrm{~m} / 11 \mathrm{f}$ & $4 \mathrm{~m} / 1 \mathrm{f}$ & $12 \mathrm{~m} / 12 \mathrm{f}$ \\
Age [years] & $53.4( \pm 21.9)$ & $9.6( \pm 4.22)$ & $44.3( \pm 13.78)$ \\
F/U [months] & $54.5( \pm 55.42)$ & & \\
\hline
\end{tabular}

\section{Case example 1}

An 11-year-old boy presented four months after previous surgery for a cervical tumor. The primary tentative diagnosis and diagnostic regime is unknown. Presumably, a complete resection of the suspected tumor was planned via a posterior approach. In the end, a tumor debulking and partial laminectomy of $\mathrm{C} 3$ and $\mathrm{C} 4$ on the left side was performed. Pathological examination revealed the tumor to be a chordoma. Four months after the surgery, the patient presented at our department. He complained of a progressive tickle in the throat and mild pain. MRI (Fig. 4a, b) showed a chordoma involving C2, C3, and C4 with mainly anterior growth and involvement of the hypoplastic left vertebral artery. Radiograph revealed a local C2-4 kyphosis of $23^{\circ}$ (Fig. 5). A staged posterior-anterior surgery was performed. First the posterior

Fig. 2 Postoperative radiotherapy
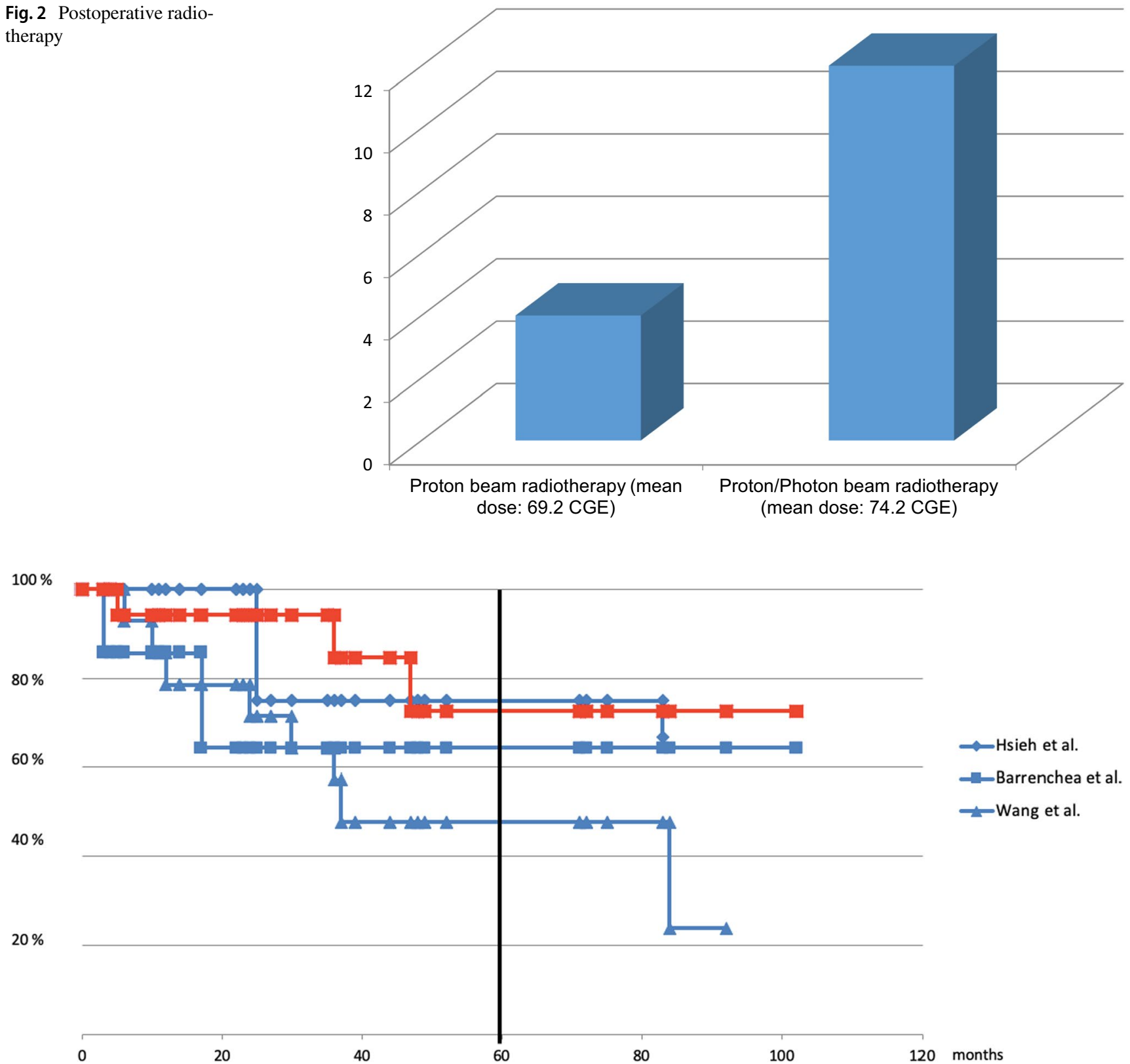

Fig. 35 -year progression-free survival. Present study compared with previously published data $[8,9,12]$ 
Fig. 4 MRI showing mainly anterior growth of the chordoma and involvement of the left vertebral artery
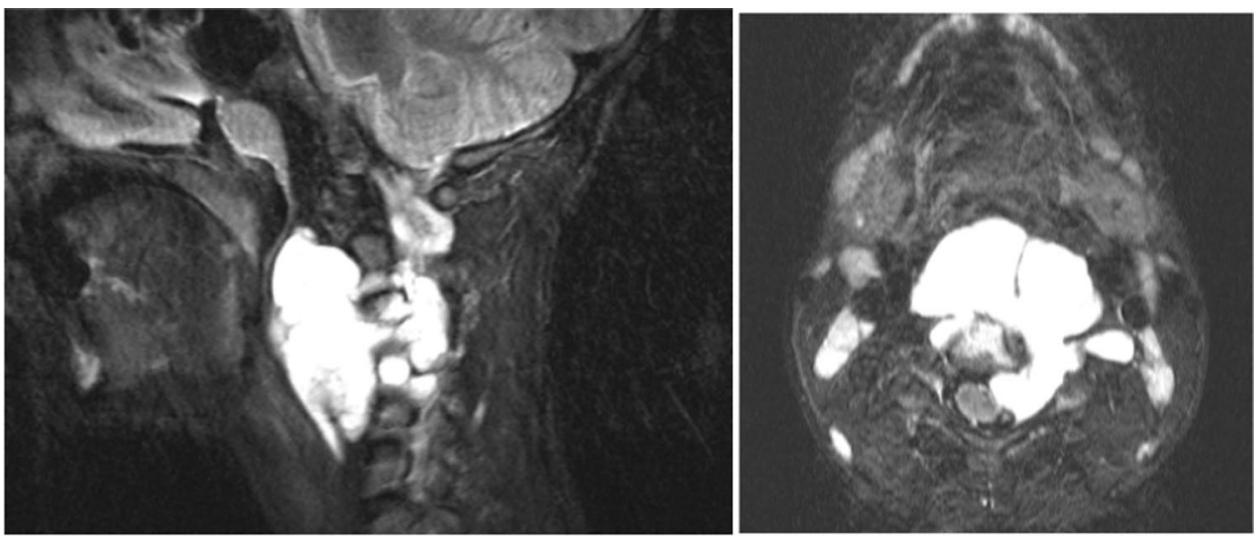

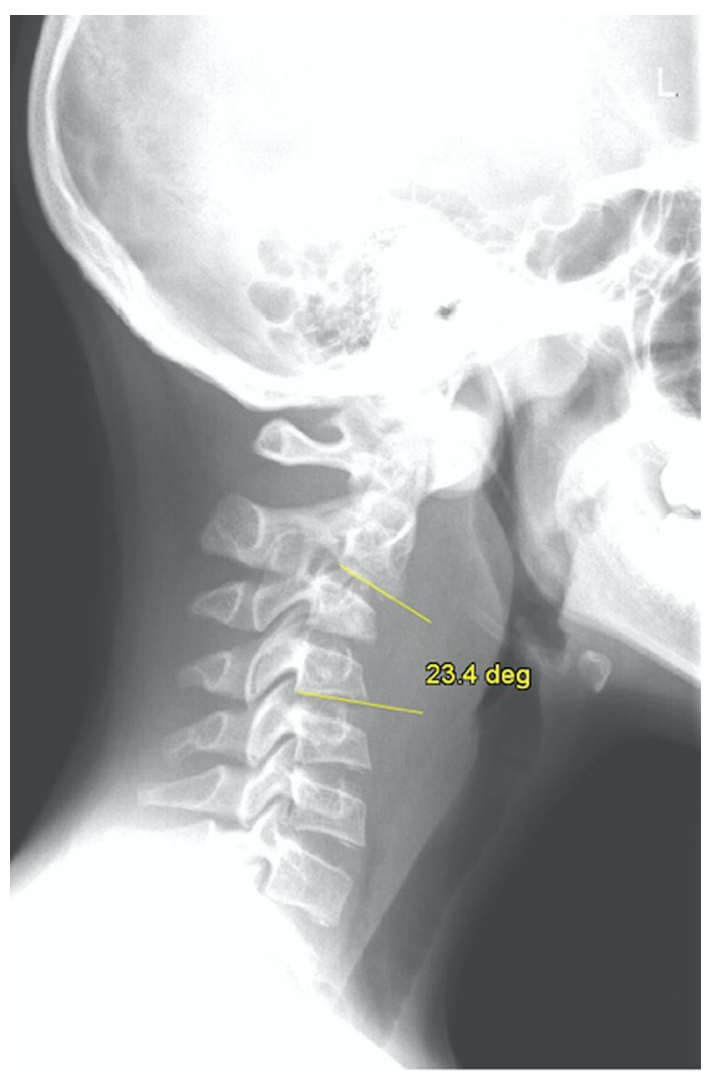

Fig. 5 Radiograph showing local kyphosis due to tumor infiltration

elements (laminae, lateral masses, and joints) of C 3 and $\mathrm{C} 4$ were completely removed on the left side. Gross total tumor resection was performed until tumor-free margins could be identified. There was no tumor involvement of the posterior elements on the right side. Posterior instrumented fusion was performed from $\mathrm{C} 2$-C5 with collagen-hydroxyapatite matrix and iliac crest bone marrow aspirate on the right side. Five days later, a gross total resection of the chordoma with complete removal of the $\mathrm{C} 3$ and $\mathrm{C} 4$ vertebral bodies and resection of the left vertebral artery was performed. The nerve roots could be preserved. An anterior spondylodesis using a bicortical iliac bone graft was performed. Postoperative radiographs show a good restoration of cervical lordosis (Fig. 6a, b). Postoperative MRI did not show any remnants of the tumor. Treatment was finished with postoperative proton beam radiotherapy. At 10-year follow-up, there is no sign of tumor recurrence.

\section{Case example 2}

A 71-year-old patient received anterior surgery for a cervical spine chordoma 13 months prior to consultation at our department. The exact type of surgery performed is unknown. Presumably, the patient received an anterior decompression and partial resection of the tumor via an anterolateral approach without spinal instrumentation. A tracheotomy was performed as well, and afterward, the patient developed a tracheal fistula. A staged posterioranterior tumor resection was planned. The preoperative MRI (Fig. 7a, b) shows a huge tumor mass. In the first step, a posterior instrumentation and fusion from occiput to $\mathrm{C} 5$ using iliac crest spongiosa was performed. Posterior elements of the spine did not show any tumor infiltration. In the second step, an anterior gross total tumor resection was performed including resection of the dens axis and $\mathrm{C} 2-4$ vertebral bodies via a bilateral high anterolateral approach. Anterior stabilization was achieved using a C2-prosthesis. The postoperative MRI revealed a small tumor remnant at the caudal aspect of the clivus (Fig. 8a, b). The tumor board decided to initiate postoperative proton beam therapy. Regular follow-up MRI did not show any sign of progression. Radiograph after four years shows solid fusion and no signs of implant failure (Fig. 9a, b).

\section{Discussion}

In a systematic literature review, Bakker et al. identified several adverse prognostic factors for progression-free and overall survival [3]. They identified non-influenceable factors: 
Fig. 6 Radiographs at five years post-op showing correction of the kyphosis and solid fusion
Fig. 7 MRI 13 months after initial surgery showing a huge tumor mass with high anterolateral growth
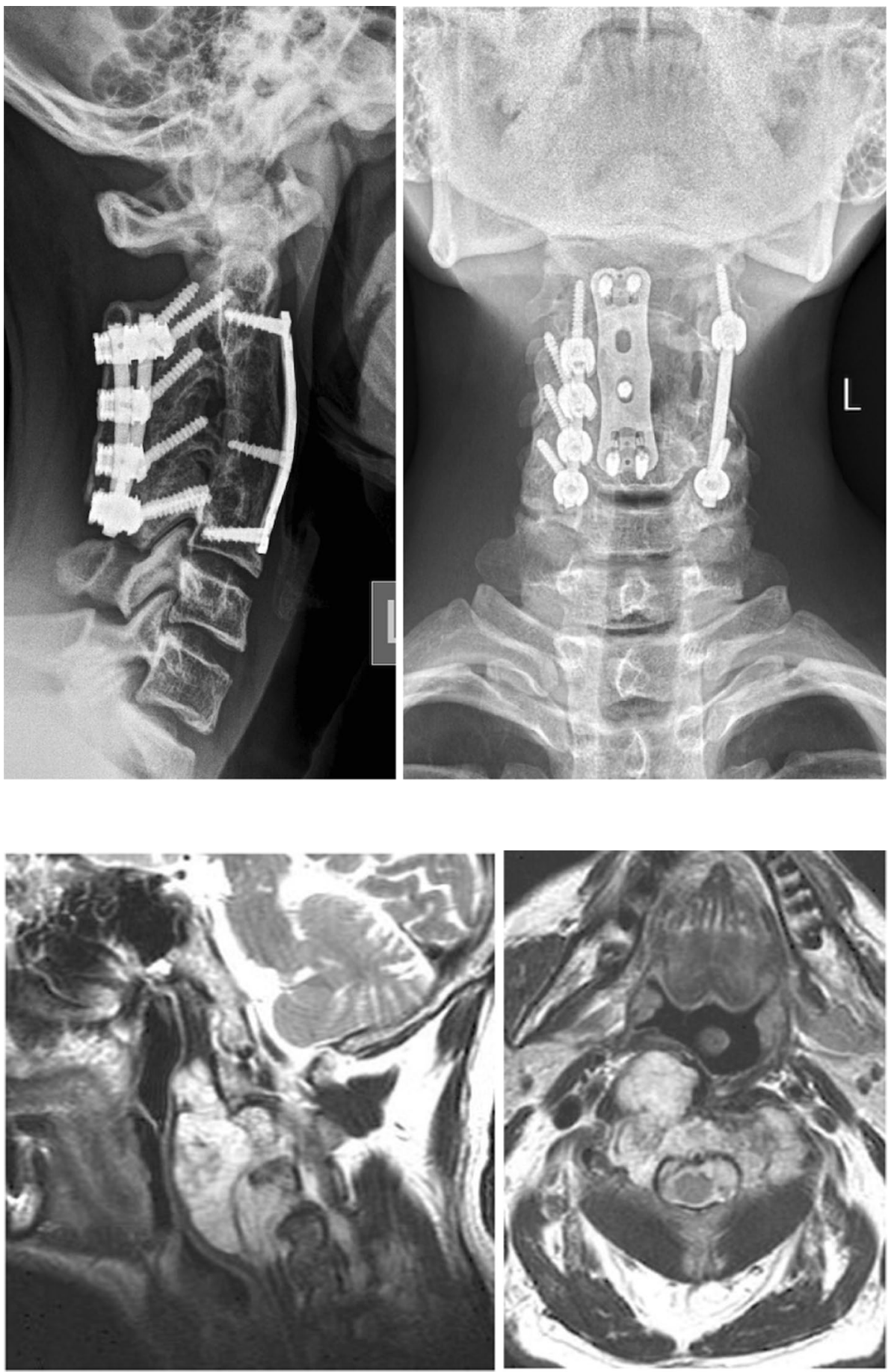

a relatively bigger tumor, dedifferentiated subtype, tumor localization at C1-C2 or S1-S5, older age, earlier year of diagnosis, presence of metastasis, and Karnofsky Performance Score $<80$. Influenceable prognostic factors were: inadequate surgical margins, no adjuvant radiotherapy, a history of prior treatment or an invasive diagnostic procedure performed at another hospital, and complications after surgery. Unfortunately, in a revision scenario after previous surgery, all influenceable factors for worse outcome apply.
Carpentier et al. pointed out the value of aggressiveness of primary surgery for complete removal of the tumor. They compared patients who underwent primary surgery with complete resection of the chordoma to patients who were treated for recurrence and found worse outcome in the latter group [18]. In a large series of 155 patients with spinal chordoma and chondrosarcoma, Murray et al. found a significantly worse outcome after incomplete tumor removal, but they did not find a superior outcome after en bloc resection 
Fig. 8 Postoperative MRI revealing a tumor remnant at the caudal aspect of the clivus
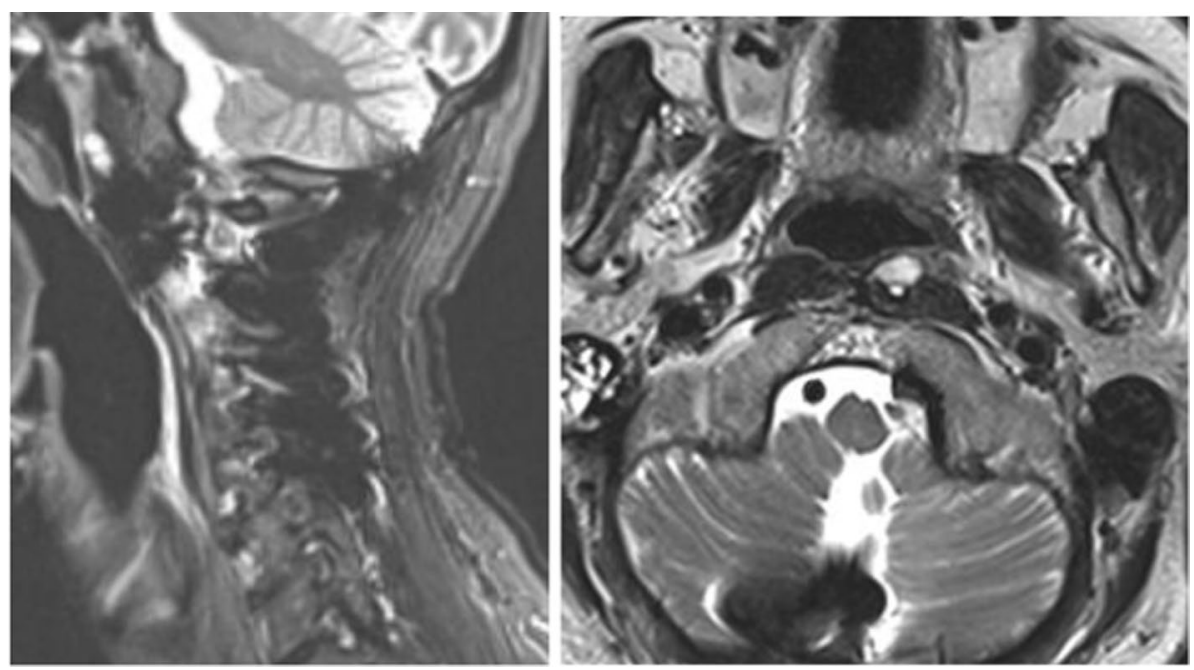

Fig. 9 Radiographs four years postoperatively
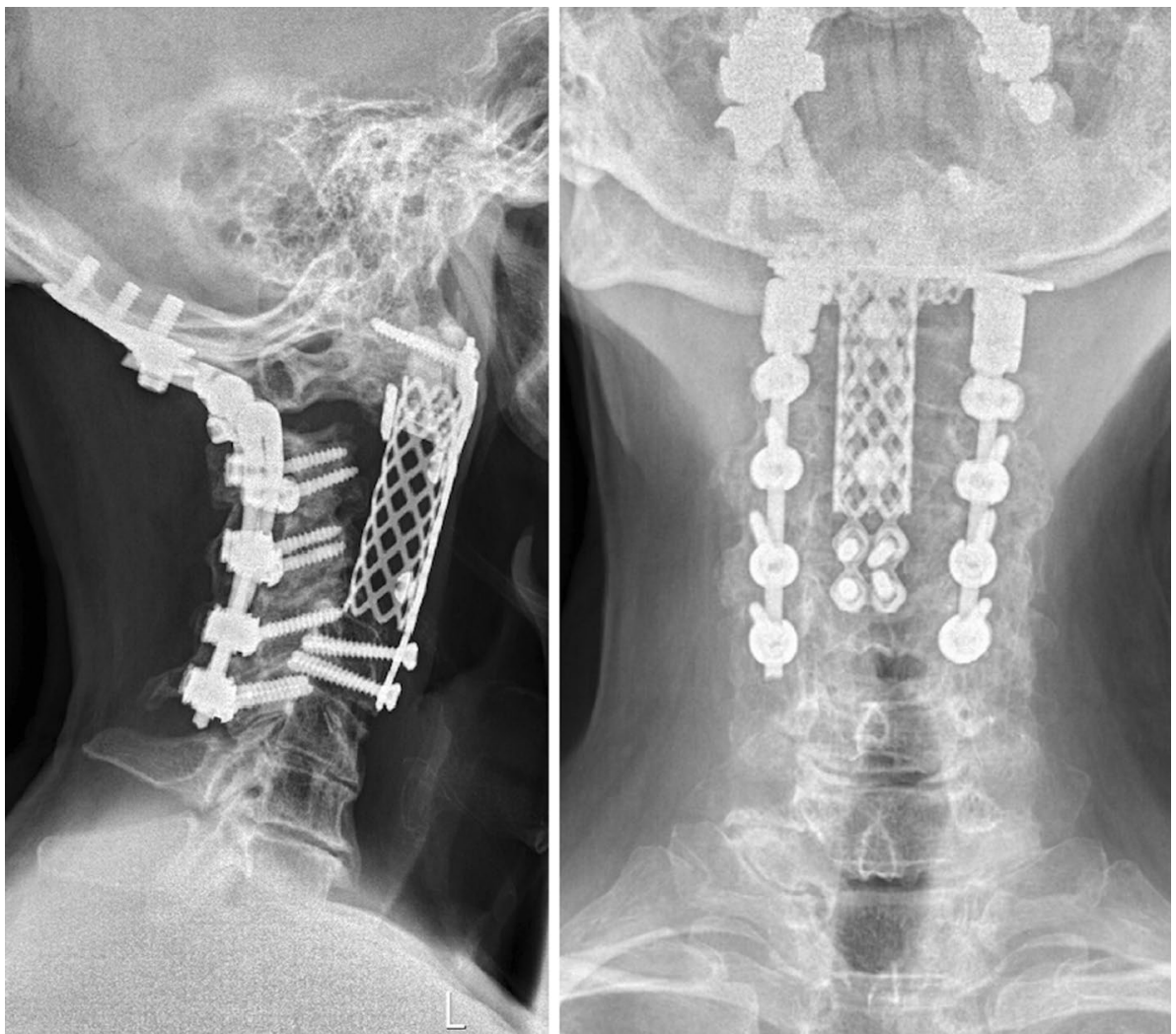

as compared to non-en bloc gross total resection but a higher complication rate in patients operated on with the intent of en bloc resection [19].

With this study, we would like to present our results after revision surgery for remnants or recurrence of cervical spine chordoma. In our cohort, we were able to achieve satisfactory results that are comparable to results after primary surgery. In the following, we would like to discuss our treatment philosophy and experiences made during treatment of these cases. We recommend to evaluate complete tumor excision by magnetic resonance imaging within $48 \mathrm{~h}$ postoperatively. In case of incomplete tumor resection, early revision surgery should be performed, if possible. With early revision surgery, extensive scaring can be avoided. Additionally, if the previous surgery is not too long ago, one may be able to remember anatomical characteristics of the individual patient a lot better than after a few weeks.

Chordomas may be excised by anterior midline approaches, transoral and transmandibular approaches, (high) anterolateral and lateral approaches, posterior 
midline approaches or any combination of these. The chosen approach depended on the exact position of the tumor, type and extent of previous surgeries. High anterolateral and lateral approaches can provide exposure of the clivus, unhindered by cranial nerves or major blood vessels. Indication for transoral surgery was very strict and reserved for (local) chordoma of $\mathrm{C} 0-2$. Rather than to be dogmatic about the ideal approach, it is more important to achieve maximum tumor excision by whichever approach the surgeon is experienced with [2].

In revision surgery, we generally try to avoid transoral approaches whenever possible because of associated complications, especially wound healing problems of the pharyngeal wall. High anterolateral approaches are usually sufficient, except for tumors located at the upper craniocervical junction. Transoral approaches are sufficient for tumors located close to the midline as the extent of lateral exposure is limited. With this approach the inferior part of the clivus down to the $\mathrm{C} 2$ vertebral body can be reached. A tracheostomy is performed when the operation involves the high nasopharynx and the craniocervical junction. Extensive transmaxillary or transmandibular approaches were not used in our collective, but can be necessary if the tumor is located too laterally from the midline. To achieve a gross total resection, it is very important to estimate how much of the tumor can be resected using which approach. This requires the surgeon to know his limits and capabilities. Help from other disciplines (e.g., maxillofacial surgery) may help to make surgery easier and can provide a more relaxed atmosphere.

In most cases, a combined anterior and posterior approach and instrumentation is necessary. Generally, it is preferable to start with the posterior approach, since the anatomy is less complex and posterior instrumentation provides stability during anterior tumor resection. For long-term stability, it is necessary to achieve bony spondylodesis, ideally using autologous bone. Any instrumentation will fail in the long term without a solid bony fusion.

In case of involvement of a dominant VA, it is mandatory to preserve this VA. In case of involvement of a co-dominant or non-dominant VA, it can be resected. We prefer to ligate the VA intraoperatively and do not use any coils preoperatively. However, resection of a VA is not mandatory if tumor tissue can be dissected from the VA and gross total tumor resection can be achieved. If tumor tissue is firmly attached to a dominant VA, it may not be possible to dissect all tumor tissue from the VA. Therefore, we generally recommend superficial bipolar electric cauterization of all tissue that had contact to the tumor, including the dura and VAs.

Although instability at the craniocervical junction can be potentially life-threatening, one can only address a tumor properly in terms of complete resection if one can achieve a biomechanically stable situation after resection. Tumor surgery should not make compromises because of stability concerns, because even multilevel defects of the cervical spine can be reconstructed. In our collective, we did not observe implant failure or implant-related instability.

An adjuvant radiotherapy is mandatory [14]. The effect of proton or carbon ion radiotherapy can be crucial for the individual patient, especially after incomplete tumor removal. However, radiotherapy should only be initiated when an appropriate surgical treatment is definitely finished, since preoperative radiotherapy is a well-documented risk factor for postsurgical complications [20, 21].

\section{Limitations}

As in most mobile spine chordoma studies, we can only report on a relatively small number of cases with partly incomplete preoperative and follow-up data. However, to our knowledge, this is to date the largest series on revision surgery in cervical spine chordoma.

Tumor biology plays an important role in local growth, aggressiveness and metastatic spread. Although differences in survival are mainly influenced by the extent of tumor resection, some differences may also be explained by different expressions of chordoma genes. There is a greater chance of recurrence in patients with an increased tumor expression of human telomerase reverse transcriptase mRNA and mutation of p53 protein [22, 23]. The Ki67 proliferation index might also predict outcome [24].

\section{Conclusion}

The principle goal of treatment should be a complete tumor resection at the time of the first presentation of the patient since complete tumor removal is the key factor for long-term survival $[2,10,18,25,26]$. Complete tumor removal is feasible in patients that underwent previous surgery, even at the craniocervical junction, which is especially challenging due to its peculiar complex anatomy. With profound preoperative planning, appropriate surgical techniques, and adjuvant postoperative radiotherapy, outcomes similar to those in primary surgery can be achieved.

Funding Open Access funding enabled and organized by Projekt DEAL. No funding was received for this study.

\section{Declarations}

Conflict of interest None of the authors has any potential conflict of interest to declare.

Open Access This article is licensed under a Creative Commons Attribution 4.0 International License, which permits use, sharing, adaptation, distribution and reproduction in any medium or format, as long as you give appropriate credit to the original author(s) and the source, 
provide a link to the Creative Commons licence, and indicate if changes were made. The images or other third party material in this article are included in the article's Creative Commons licence, unless indicated otherwise in a credit line to the material. If material is not included in the article's Creative Commons licence and your intended use is not permitted by statutory regulation or exceeds the permitted use, you will need to obtain permission directly from the copyright holder. To view a copy of this licence, visit http://creativecommons.org/licenses/by/4.0/.

\section{References}

1. Eriksson B, Gunterberg B, Kindblom LG (1981) Chordoma. A clinicopathologic and prognostic study of a Swedish national series. Acta Orthop Scand 52:49-58

2. Choi D, Gleeson M (2010) Surgery for chordomas of the craniocervical junction: Lessons learned. Skull Base 20:41-45

3. Bakker SH, Jacobs WCH, Pondaag W et al (2018) Chordoma: a systematic review of the epidemiology and clinical prognostic factors predicting progression-free and overall survival. Eur Spine J 27:3043-3058. https://doi.org/10.1007/s00586-018-5764-0

4. Flanagan A, Yamaguchi T (2013) Chordoma. In: Fletcher C, Bridge J, CW P, Mertens F (eds) World Heal. Organ. Classif. tumours soft tissue bone. Pathol. Genet. pp 328-9

5. Boriani S, Chevalley F, Weinstein JN, et al. (1996) Chordoma of the spine above the sacrum. Treatment and outcome in 21 cases. Spine (Phila Pa 1976) 21:1569-77.

6. Bergh P, Kindblom LG, Gunterberg B et al (2000) Prognostic factors in chordoma of the sacrum and mobile spine.pdf. Cancer 88:2122-2134

7. Aoun SG, Elguindy M, Barrie U et al (2018) Four-level vertebrectomy for en bloc resection of a cervical chordoma. World Neurosurg 118:316-323. https://doi.org/10.1016/j.wneu.2018.07. 153

8. Hsieh PC, Gallia GL, Sciubba DM, et al. (2011) En bloc excisions of chordomas in the cervical spine: review of five consecutive cases with more than 4-year follow-up. Spine (Phila Pa 1976) 36:E1581-7.

9. Barrenechea IJ, Perin NI, Triana A et al (2007) Surgical management of chordomas of the cervical spine. J Neurosurg Spine 6:398-406

10. Choi D, Melcher R, Harms J, Crockard A (2010) Outcome of 132 operations in 97 patients with chordomas of the craniocervical junction and upper cervical spine. Neurosurgery 66:59-65; discussion 65.

11. Molina CA, Ames CP, Chou D et al (2014) Outcomes following attempted en bloc resection of cervical chordomas in the C- 1 and $\mathrm{C}-2$ region versus the subaxial region: a multiinstitutional experience. J Neurosurg Spine 21:348-356

12. Wang Y, Xiao J, Wu Z et al (2012) Primary chordomas of the cervical spine: A consecutive series of 14 surgically managed cases: Clinical article. J Neurosurg Spine 17:292-299. https://doi. org/10.3171/2012.7.SPINE12175
13. Yasuda M, Bresson D, Chibbaro S, et al. (2012) Chordomas of the skull base and cervical spine: clinical outcomes associated with a multimodal surgical resection combined with proton-beam radiation in 40 patients. Neurosurg Rev 35:171-82; discussion 182-3.

14. Stacchiotti S, Sommer J (2015) Building a global consensus approach to chordoma: a position paper from the medical and patient community. Lancet Oncol 16:e71-e83

15. Ergun O, Gunes Tatar I, Birgi E, Hekimoglu B (2016) Evaluation of vertebral artery dominance, hypoplasia and variations in the origin: Angiographic study in 254 patients. Folia Morphol 75:33-37. https://doi.org/10.5603/FM.a2015.0061

16. Jeszenszky D, Fekete TF, Melcher R, Harms J (2007) C2 prosthesis: anterior upper cervical fixation device to reconstruct the second cervical vertebra. Eur Spine J 16:1695-1700

17. Crockard HA, Steel T, Plowman N et al (2001) A multidisciplinary team approach to skull base chordomas. J Neurosurg 95:175-183

18. Carpentier A, Polivka M, Blanquet A et al (2002) Suboccipital and cervical chordomas: the value of aggressive treatment at first presentation of the disease. J Neurosurg 97:1070-1077

19. Murray FR, Snider JW, Schneider RA et al (2020) Prognostic factors for spinal chordomas and chondrosarcomas treated with postoperative pencil-beam scanning proton therapy: a large, single-institution experience. J Neurosurg Spine. https://doi.org/10. 3171/2019.11.SPINE1927

20. Marks JE, Freeman RB, Lee F, Ogura JH Pharyngeal wall cancer: an analysis of treatment results complications and patterns of failure. Int J Radiat Oncol Biol Phys 4:587-93.

21. Weber RS, Berkey BA, Forastiere A et al (2003) Outcome of salvage total laryngectomy following organ preservation therapy: the radiation therapy oncology group trial 91-11. Arch Otolaryngol Head Neck Surg 129:44-49

22. Pallini R, Maira G, Pierconti F et al (2003) Chordoma of the skull base: predictors of tumor recurrence. J Neurosurg 98:812-822

23. O'Connell JX, Renard LG, Liebsch NJ et al (1994) Base of skull chordoma. A correlative study of histologic and clinical features of 62 cases. Cancer 74:2261-2267

24. Holton JL, Steel T, Luxsuwong M et al (2000) Skull base chordomas: correlation of tumour doubling time with age, mitosis and Ki67 proliferation index. Neuropathol Appl Neurobiol 26:497-503

25. Boriani S, Saravanja D, Yamada Y, et al. (2009) Challenges of local recurrence and cure in low grade malignant tumors of the spine. Spine (Phila Pa 1976) 34:S48-57.

26. Wang Y, Xu W, Yang X, et al. (2013) Recurrent upper cervical chordomas after radiotherapy: surgical outcomes and surgical approach selection based on complications. Spine (Phila Pa 1976) 38:E1141-8.

Publisher's Note Springer Nature remains neutral with regard to jurisdictional claims in published maps and institutional affiliations. 